\title{
Endpoint Matrix: a Conceptual Tool to Promote Consideration of the Multiple Dimensions of Humane Endpoints
}

\section{Vanessa Ashall and Kate Millar}

Centre for Applied Bioethics, School of Biosciences and School of Veterinary Medicine and Science, University of Nottingham, Sutton Bonington, UK

\begin{abstract}
Summary
This paper proposes a framework to support appropriate application of endpoints in animal experiments. It is recommended that unpredicted endpoints should be explicitly considered alongside scientific endpoints and justifiable endpoints as the three types of endpoint which comprise the "humane." We suggest there is a need for clear identification of each type of endpoint and an understanding of the interactions between these types. The use of an "endpoint matrix" during study planning is proposed to promote methodically sound and consistent definition, determination, and detection of unpredicted, scientific, and justifiable endpoints in animal experiments. It is claimed that the further development and use of this tool will support a more effective and harmonized practical application of humane endpoints for all animal use in line with best practice recommendations.
\end{abstract}

Keywords: humane endpoint, scientific endpoint, justifiable endpoint, unpredicted endpoint, endpoint matrix

\section{Introduction}

The most widely used definition of humane endpoints, published by the OECD (2000: p. 10), sets out humane endpoints as: "The earliest indicator in an animal experiment of severe pain, severe distress, suffering or impending death." However, humane endpoints have been recently described as "a refinement strategy designed to minimise pain, suffering or distress experienced by animals during an experiment" (Hendriksen et al., 2011: p. 344). This more recent description reflects the progress that has been made over the last fifteen years in the drive to prevent avoidable laboratory animal suffering, through recognizing humane endpoints as a vital component in the application of the "Three Rs" principles. Standards in this area have also been embedded within the laws that govern animal use; the phrase "humane endpoint" was first recognized legally across the EU on January 1, 2013 when member states were required to implement Directive 2010/63/EU (EU, 2010) on the protection of animals used for scientific purposes into national law. However, we have questioned the construction of humane endpoints within this legislation (Ashall and Millar, 2013), with suggestions that the Directive is reliant on the narrow definition of humane endpoints proposed by OECD over 10 years ago $(\mathrm{OECD}, 2000)$ which promotes humane endpoints as an alternative to death as an endpoint. We (Ashall and Millar, 2013) have expressed a concern that the Directive will not promote recent advances in understanding and best practice application (Hendriksen et al., 2011) of this concept which presents humane endpoints as a refinement strategy designed to minimize suffering and eliminate any avoidable suffering in all animal experiments.

Using examples of practical tools designed to aid ethical reflection and decision-making (e.g., Lindl et al., 2012; Mepham, 1996; England and Millar, 2008), it is proposed here that there is a need to formally structure the detailed ethical analysis and practical decision-making required in fully embracing this important refinement strategy. Initially, best practice guidance on the application of humane endpoints is discussed. Hendriksen et al. (2011) articulate the complexity of this concept and we extend this work to suggest that there exist several different types of experimental endpoints which are important for the articulation of "humane."

We propose that the concept of "unpredicted" should be considered alongside "scientific" and "justifiable" when determining endpoints, with each requiring individual consideration alongside an understanding of their interactions. Through the development of an endpoint matrix we provide a format for the consideration of each of these three types of endpoint, suggesting that:

a) Each type of endpoint is accurately defined;

b) The practical application of each endpoint to the experiment is determined; and

c) Information is collected which will allow endpoints to be accurately detected.

It is suggested that the further development and use of the endpoint matrix will result in the appropriate selection of the most humane endpoint and should reduce overall animal suffering through more effective use of this refinement strategy. 


\section{Classes of humane endpoints}

Humane endpoints have long been recognized as important in reducing avoidable laboratory animal suffering. The term has been commonly used in published literature since a conference held in 1998 in Zeist, entitled "Humane Endpoints in Animal Experiments for Biomedical Research" (Hendriksen and Morton, 1999). In the following years a number of important publications addressed this concept, including international guidelines on their practical use from the Canadian Council on Animal Care (1998) and the Organisation for Economic Cooperation and Development (2000). More recent publications have challenged researchers to consider how humane are traditional practical approaches to humane endpoints (Franco et al., 2012) and recent best practice guidance has built on these important earlier works to develop a concept of humane endpoints which can be a powerful refinement strategy (Hendriksen et al., 2011). We have suggested (Ashall and Millar, 2013) that this recent practical guidance positively reflects the conceptual complexity of humane endpoints and thus can demand greater reflection from a researcher than traditional near-death endpoints. To illustrate this point, Hendriksen et al. (2011: p. 345) suggest that "there are four specific situations in which the application of humane endpoints should be considered." These situations are summarized below as defined by Hendriksen et al. (2011):

1. When the scientific objectives have been met and there is no reason to continue the investigation.

2. When unexpected suffering occurs. In this situation, the suffering is not related to the experiment but is unexpected and has not been anticipated at the outset.

3. When suffering was anticipated at the start of the experiment, but has become more severe than predicted.

4. When pain and/or distress are an inherent part of the experiment and are anticipated at the outset.

This conceptual framing could be extended as follows: the different situations presented above relate to different types of endpoint, each having implications for the determination of "humane." Some of these have been individually characterized in the literature and as such they are elaborated below.

A. Scientific endpoint which relates to situation 1 above has previously been defined as "the criteria that will be used to indicate that the experimental objective has been reached" (Wallace, 2000). This endpoint does not relate to animal measures of suffering but rather to the scientific or other data which must be gathered to achieve the objectives of an experiment. As further scientific information is not required, any suffering (whether severe or not) caused when an experiment continues beyond this point would be considered "avoidable." Its use as an endpoint is humane because this represents the earliest point at which the experiment can be ended without data loss. However, an experiment will only be ethically acceptable within a cost/benefit calculus when the scientific endpoint proposed falls within the limits of permissible suffering.

B. Justifiable endpoint: The identification of humane endpoints through cost-benefit justification in animal experiments (situation 4 above) has recently been addressed following the drafting of Directive 2010/63/EU. Lindl et al. (2012) suggest that " $a$ justifiable balance is required between the needs of humans, animals or the environment and the pain, suffering, and distress of animals proposed to meet these needs." We propose the use of an original term, i.e., "justifiable endpoint," to define humane endpoints which are based upon a cost/benefit justification. As part of the ethical approval of experiments expected to cause any pain or suffering, the benefits expected to be achieved must be used to justify a specified level of suffering. The justifiable endpoint is reached once this level of pain or suffering is detected. As it is not considered ethically justifiable to exceed this endpoint, it could be considered the absolute limit of "humane" experimentation. Hendriksen et al. (2011) highlight the important interaction between the scientific endpoint and a humane endpoint identified during cost/benefit justification: if the scientific endpoint cannot be reliably reached before the justifiable endpoint, animals will be removed from the study before the experimental aims are achieved, resulting in data loss and therefore unjustifiable animal use.

We propose here that these two previously identified humane endpoints do not encompass a clear consideration of unexpected outcomes (Hendriksen et al., 2011, situations 2 and 3). Unexpected suffering can occur during an experiment, either due to changes which are not directly related to the research experiment or to suffering which is different from that expected as a result of the experiment. We suggest that "unpredicted endpoints", which have not previously been explicitly defined, also need to be reflected upon and planned for.

C. Unpredicted endpoint: Unexpected suffering might occur following an unrelated illness or accident or from unexpected adverse effects of the research. Unexpected suffering might necessitate humane intervention, irrespective of whether the scientific or justifiable endpoint has been reached, since the suffering is not related to the attainment of the scientific goal or has not been factored into a costbenefit justification for the study. This type of suffering presents a greater challenge to the research team since its form and severity cannot be factored into the cost-benefit justification during research planning and therefore this type of endpoint cannot be defined in advance. It is very important, however, that the management of unpredicted endpoints is carefully considered so responsive plans are defined as part of study design. This means it is essential that animals are regularly monitored for general signs of pain and suffering in addition to any specific expected signs. When expected suffering is occurring, it should be regularly determined that additional adverse events are not also occurring. Since the level of suffering justified by the expected benefits of the experiment cannot be exceeded, in such a scenario we suggest that the concept of "cumulative suffering" is important. This concept has previously been applied to cumulative measurements of the severity or duration of suffering in experimental animals (Honess and Wolfensohn, 2010). Humane intervention will be required if the suffering caused by adverse events alone or in 
addition to any expected suffering exceeds that which was justified by the expected benefits of the study. In practice this would mean that animals suffering due to unpredicted adverse events should not simultaneously be exposed to the levels of expected suffering justified in the cost/benefit calculus. Unless adverse events can be managed to result in minimal suffering or the scientific endpoint can be achieved well before the identified justifiable endpoint, adverse events might therefore lead to humane intervention before the scientific endpoint is achieved.
We have explored unpredicted endpoints as an important type of humane endpoint which should be considered in the effective application of this refinement strategy. We suggest that each of these three types of humane endpoint should be both individually and jointly considered during study design and throughout the research process. As illustrated above, the endpoint which is ultimately defined as humane will require careful consideration on every occasion. The endpoint matrix (Tab. 1) was designed to facilitate methodical consideration of each of these ethical endpoints.

\section{Tab. 1: The endpoint matrix}

The endpoint matrix is envisaged for initial use as a blank document which can help structure the necessary reflective processes during the planning stage of each study. Here the matrix contains example data translations for each cell to illustrate some important considerations. It will need to be considered in several stages and by different personnel; as such the tool should provide an opportunity for reflective and efficient team communication. Once completed the matrix should be used as a reference document during the study to accurately alert staff to the achievement of an endpoint. After the study the matrix can be kept as a record of the endpoints which were reached and reviewed in dialogue with the research team, a form of research debriefing, to help improve research protocols and communication strategies. It may be useful also for retrospective review, encouraging further progress to be made in the application of humane endpoints.

\begin{tabular}{|c|c|c|c|}
\hline & $\begin{array}{l}\text { Define } \\
\text { What is it? }\end{array}$ & $\begin{array}{l}\text { Determine } \\
\text { How does it apply to the experiment? }\end{array}$ & $\begin{array}{l}\text { Detect } \\
\text { Who, how, and when? }\end{array}$ \\
\hline Scientific endpoint & $\begin{array}{l}\text { The criteria used to indicate } \\
\text { the experimental objective has } \\
\text { been reached. }\end{array}$ & $\begin{array}{l}\text { What specific and minimum } \\
\text { (e.g., } \mathrm{P}<0.05) \text { data is required? } \\
\text { At what point will no further data } \\
\text { be required? } \\
\text { How does this affect expected } \\
\text { suffering and cost benefit } \\
\text { justification? }\end{array}$ & $\begin{array}{l}\text { Who will set the scientific } \\
\text { endpoint? (e.g., PI or } \\
\text { responsible investigator). } \\
\text { How and when will data } \\
\text { collection be monitored and } \\
\text { how will this be reported? } \\
\text { Who will determine when } \\
\text { the scientific endpoint has } \\
\text { been reached? }\end{array}$ \\
\hline Justifiable endpoint & $\begin{array}{l}\text { The maximum level of suffering } \\
\text { which can be justified by } \\
\text { the expected benefits of the } \\
\text { experiment. } \\
\text { This degree of suffering will } \\
\text { necessitate the ending of } \\
\text { the experiment, even if the } \\
\text { scientific endpoint has not yet } \\
\text { been achieved. }\end{array}$ & $\begin{array}{l}\text { Ethical review should perform a } \\
\text { cost/benefit analysis of studies } \\
\text { which are expected to cause } \\
\text { suffering. } \\
\text { The animal indicators of the limit } \\
\text { of justifiable suffering should } \\
\text { be determined before the study } \\
\text { commences. } \\
\text { How could any expected suffering } \\
\text { be avoided, alleviated and/or } \\
\text { minimized? }\end{array}$ & $\begin{array}{l}\text { Who is trained to recognize } \\
\text { expected suffering? } \\
\text { How will they recognize and } \\
\text { report the justifiable endpoint? } \\
\text { Who will decide to end the } \\
\text { experiment? } \\
\text { What action should be taken and } \\
\text { what alternatives are available? }\end{array}$ \\
\hline Justifiable endpoint & $\begin{array}{l}\text { An endpoint identified by } \\
\text { unexpected suffering which is } \\
\text { not related to the experimental } \\
\text { aims or is different from that } \\
\text { which was expected. }\end{array}$ & $\begin{array}{l}\text { General indicators of pain and/ } \\
\text { or suffering must be monitored in } \\
\text { addition to expected specific signs. } \\
\text { The experiment must be ended } \\
\text { if unexpected events occur which, } \\
\text { when considered alongside ex- } \\
\text { pected suffering, result in cumulative } \\
\text { suffering beyond that justified } \\
\text { by cost benefit or if unexpected } \\
\text { events are likely to interfere with } \\
\text { the achievement of the scientific } \\
\text { endpoint }\end{array}$ & $\begin{array}{l}\text { Who is trained to detect } \\
\text { unexpected pain and suffering? } \\
\text { Who will determine whether } \\
\text { the experiment should continue } \\
\text { (e.g., designated vet and animal } \\
\text { welfare officer will need to define } \\
\text { cumulative suffering and consider } \\
\text { this alongside the agreed } \\
\text { justifiable endpoint)? } \\
\text { What action should be taken } \\
\text { and what alternatives are } \\
\text { available? }\end{array}$ \\
\hline
\end{tabular}




\section{Endpoint matrix}

The development of tools which help to guide or structure ethical reflection is not a new approach. Within the context of animal experimentation and in response to Directive 2010/63/ EU, Lindl et al. (2012) recently proposed a formal procedure which aims to ensure the ethical defensibility of applications for animal experiments by balancing potential benefits against harm to the experimental animals. This is one recent example of the publication of practical guidance which aims to support structured and more transparent ethical assessment. A further example of this type of tool is the "ethical matrix" (Mepham, 1996) which was originally developed for examining the ethics of animal biotechnology development and application, and likewise aims to facilitate ethical analysis. This tool has subsequently been developed and applied in a number of different ways, potentially exemplifying the need for ethical tools to support decision-making in the context of the complex set of ethical dilemmas that scientists and others working with animals face (England and Millar, 2008; Millar, 2011). Thus we propose there is a need to develop a practice-oriented tool, such as those discussed above, to facilitate the comprehensive application of this complex refinement strategy; guiding researchers through the ethical and practice-related decisions involved.

With reference to the "four situations" identified above, Hendriksen et al. (2011) suggest that "each situation requires a specific decision to be made with regard to the application of humane endpoints." Our description of the three types of endpoint above and published literature further illustrates this point; scientific endpoints can be detected through data collected by the research scientists whilst justifiable endpoints and unpredicted endpoints will require the detection of suffering by animal care staff working with the research scientists (Morton, 2000). Justifiable endpoints can be accurately defined during experimental planning whereas unpredicted endpoints should be discussed in terms of management strategies, but they are determined during the experiment. There is consequently variation in the type of information required, timescales, individual staff responsibilities, and a need for effective communication and coordination within the research team. The need to apply these data to each type of endpoint has led us to develop a tabular matrix which encourages researchers to define each type of endpoint, determine how they might apply to a particular experiment, and identify how each will be accurately detected during the study (Tab. 1). It is important to recognize that this format is not intended as an indication that each endpoint can be considered in isolation. The determination of scientific and justifiable endpoints is intrinsically linked because a particular scientific goal is used to justify expected suffering, however, detection of either endpoint would be likely to require humane intervention. Likewise, adverse events occurring alone or in addition to expected suffering may result in an unpredicted endpoint being achieved before the scientific endpoint is met.

\section{Discussion}

We have presented unpredicted endpoints as an additional factor which needs to be explicitly considered alongside scientific endpoints and justifiable endpoints in aiming to achieve the most humane endpoint in animal experiments.

The development of the endpoint matrix represents an original framework for unpacking humane endpoints in animal experimentation; it further develops the notion of "humane" as defined through three different types of endpoint. Furthermore, it provides a conceptual framework for structuring the information and decision-making necessary to practically implement this complex refinement strategy. It has historically been the case that the relativity of the term "humane" has led to changes in the application of humane endpoints as we continue to strive to make progress in the important commitment to the 3Rs (Ashall and Millar, 2013). The endpoint matrix is presented here as a conceptual tool to help structure reflective processes, the next development step is to operationalize the matrix through scenario testing in order to support the accurate practical application of the most humane experimental endpoints.

\section{References}

Ashall, V. and Millar, K. (2013). An opportunity to refocus on the 'humane' in experimental endpoints: Moving beyond Directive 2010/63/EU. Altern Lab Anim 41, 307-312. http://www. frame.org.uk/atla_article.php?art_id=1551\&abstract=true (accessed 31.01.14)

Canadian Council on Animal Care (1998). Guidelines on choosing an appropriate endpoint in experiments using animals for research, teaching and testing (30pp). Ottowa, Ontario, Canada. http://www.ccac.ca/Documents/Standards/Guidelines/ Appropriate_endpoint.pdf (accessed 08.05.13)

England, G. and Millar, K. (2008). The ethics and role of AI with fresh and frozen semen in dogs. Reprod Domest Anim 43, Suppl 2, 165-171. http://dx.doi.org/10.1111/j.1439-0531. 2008.01157.x

EU - European Union (2010). Directive 2010/63/EU of the European Parliament and of the Council of 22 September 2010 on the protection of animals used for scientific purposes. Official J EU L 276, 33-79. http://eur-lex.europa.eu/LexUriServ/ LexUriServ.do?uri=OJ:L:2010:276:0033:0079:en:PDF (accessed 08.05.13)

Franco, N. H., Correia-Neves, M., and Olsson, I. A. S. (2012). How "humane" is your endpoint? - Refining the science driven approach for termination of animal studies of chronic infection. PLoS Pathology 8, e1002399. doi:10.1371/ journal.ppat.1002399, http://www.plospathogens.org/article/ info\%3Adoi\%2F10.1371\%2Fjournal.ppat.1002399

Hendriksen, C. F. M. and Morton, D. B. (1999). Humane end points in animal experiments for biomedical research. Proceedings of the International Conference (150pp). London, UK: Royal Society of Medicine Press.

Hendriksen, C., Morton, D., and Cussler, K. (2011). Use of Hu- 
mane End Points to Minimise Suffering. In B. Howard, T. Nevalainen, and G. Perretta (ed.), The Cost Manual of Laboratory Animal Care and Use (333-353). FL, USA: CRC Press.

Honess, P. and Wolfensohn, S. (2010). The extended welfare assessment grid: A matrix for the assessment of welfare and cumulative suffering in experimental animals. Altern Lab Anim 38, 205-212. http://www.frame.org.uk/atla_article. php?art_id=1276\&abstract=true

Lindl, T., Gross, U., Ruhdel, I., et al. (2012). Guidance on determining indispensibility and balancing potential benefits of animal experiments with costs to the animals with specific consideration of EU Directive 2010/63/EU. ALTEX 29, 219-228. http://www.altex.ch/Current-issue.50.html?iid=131\&aid=9

Mepham, B. (1996). Ethical analysis of food biotechnologies: An evaluative framework. In B. Mepham (ed.), Food Ethics (101-119). London, UK: Routledge.

Millar, K. (2011). Ethics and ethical analysis in veterinary science: The development and application of the ethical matrix method. In C. M. Wathes, S. A. Corr, S. A. May, et al. (eds.), Veterinary and Animal Ethics (100-112). Chichester, UK: Wiley-Blackwell.

Morton, D. B. (2000). Humane endpoints for animals used in biomedical research and testing: A systematic approach for establishing humane endpoints. ILAR J 41, 80-86. http:// dx.doi.org/10.1093/ilar.41.2.80

OECD - Organisation for Economic Co-operation and Development (2000). OECD Guidance Document on the Recognition, Assessment and use of Clinical signs as Humane Endpoints for Experimental Animals used in Safety Evaluation. Paris, France: OECD.

Wallace, J. (2000). Humane endpoints and cancer research. ILAR J 41, 87-93. http://dx.doi.org/10.1093/ilar.41.2.87

\section{Acknowledgements}

The authors would like to acknowledge the participation of researchers from the EU Network of Animal Disease Infectiology Researchers (NADIR) and the invited speakers at a Humane Endpoints workshop held at the University of Nottingham, UK, in November 2012, which inspired this paper. This work has received funding from the European Community's Seventh Framework Programme Research Infrastructures action (FP7 2007-2013), under grant agreement No. FP7-228394 (NADIR). A report written for NADIR on the use of Humane Endpoints in Large Animal Infectious Disease Research and the NADIR workshop on this topic resulted in the identification of the themes on which this paper is based. NADIR is a large network; the views expressed in this paper are those of the authors alone, and do not necessarily reflect the views of collaborators within the consortium.

\section{Correspondence to}

Vanessa Ashall, BVSc CertWEL MRCVS

Centre for Applied Bioethics

School of Biosciences and School of

Veterinary Medicine and Science

Sutton Bonington Campus

University of Nottingham

Sutton Bonington

LE12 5RD, UK

e-mail: vanessa.ashall@nottingham.ac.uk 